\title{
Tumour tactician
}

\begin{abstract}
Neurosurgeon Harry Bulstrode at the University of Cambridge, UK, is eager to research new treatments for glioblastoma, an aggressive yet common type of brain tumour. In May, he won a £200,000 (US\$255,000) Cancer Research UK Pioneer Award to investigate whether the Zika virus, which has been linked to thousands of cases of microencephaly in newborns, offers a promising treatment pathway.
\end{abstract}

\section{What attracted you to glioblastomas?}

They are the most aggressive of primary brain tumours. Fewer than $5 \%$ of the 2,300 people diagnosed in England each year survive for 5 or more years. These tumours have rather unusual biology. They are mainly creatures of brain tissue; they don't usually spread throughout the body. All through my $\mathrm{PhD}$ programme, a recurring theme was the parallels between how glioma stem cells drive tumour development and how neural stem cells grow in fetuses. As a rule, adult brain cells don't display this rapid growth pattern. Glioblastoma tumours are the exception. My PhD work left one question unanswered - how to specifically target these tumour-causing cells.

\section{How did you get the idea to test Zika as a possible brain-tumour treatment?}

As soon as published papers confirmed that Zika caused specific damage to the developing brain while generally sparing mature cells, a light bulb went on for me. If the cancer cells resemble those in the developing brain, maybe Zika could attack them, too? If Zika could cross the blood-brain barrier and target glioma stem cells while passing by normal adult brain cells - two formidable hurdles for existing treatments - it could open up a way to use Zika to attack the tumour.

\section{Did you seek advice from colleagues?}

Yes. I dropped the idea into an e-mail to my $\mathrm{PhD}$ adviser, Steven Pollard. He said that it was an interesting idea, and reassured me that I wasn't crazy. Other mentors linked me up to a facility working with Zika and put me onto the Pioneer Award idea.

\section{Are there concerns about your Zika research?}

Pollard pointed out that any clinical trial could struggle to secure ethics approval to deliberately infect people with Zika. But I

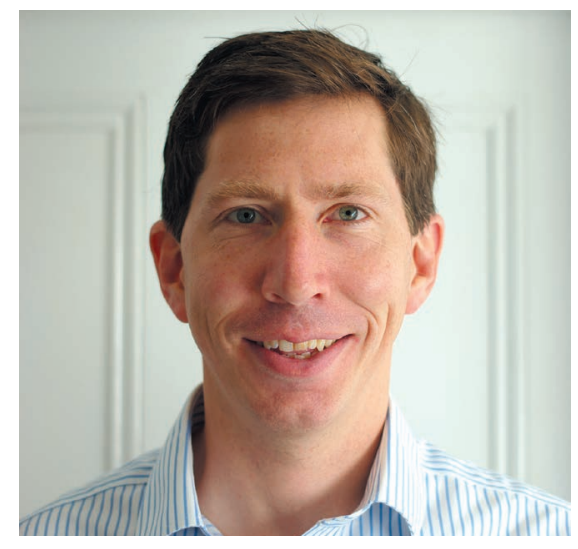

thought, why not explore the idea? Three million people have had this infection in the Americas. And adults who contracted it have almost universally made excellent recoveries or did not even notice the infection. I don't think it's a crazy idea to one day be able to offer the Zika virus or a modified version of it in a clinical trial to a person facing the prospect of death by brain tumour. But even before that step, this research offers interesting prospects for learning from Zika how to design potential therapies. A group at Cambridge recently published a study highlighting how Zika binds to a protein produced by neural stem cells, which simultaneously increases the viral turnover that leads to their destruction (P. L. Chavali et al. Science http://dx.doi. org/10.1126/science.aam9243; 2017).

\section{How would you describe the risk level for this} project?

The Pioneer Award is for high-risk projects. That said, I think this is quite a safe bet. The techniques are not revolutionary: cell cultures of tumour lines and neural stem cells are well established. The mouse models might be difficult to get right. Mice don't get Zika, so we'll need to work on how to give brain tumours and Zika to immunecompromised mice. If we can use Zika to show that tumours in the mice are smaller or ablated, that would be a huge result.

How might this project impact your career? As an MD-PhD, my hope is to offer people with tumours the option of being involved in clinical trials. I think this project should help set me up well for that.

\section{INTERVIEW BY VIRGINIA GEWIN}

This interview has been edited for length and clarity.

\section{GENDER BIAS}

Citation lag in astronomy

\author{
Female first authors' work \\ is cited less often.
}

\section{BY VIRGINIA GEWIN}

1 stronomy publications with first authors who are female receive roughly $10 \%$ fewer citations than do those with male first authors, finds a study in Nature Astronomy (N. Caplar et al. Nature Astron. 1, 0141; 2017).

In 2014, the study's lead author, Neven Caplar, a PhD student in astronomy at the Swiss Federal Institute of Technology in Zürich, attended a talk at the institute on women in science by Meg Urry, an astronomer at Yale University in New Haven, Connecticut. Inspired by Urry's presentation, Caplar and two fellow $\mathrm{PhD}$ students sought to confirm anecdotal evidence of gender bias in astronomy. They used data collated from the Astrophysics Data System, a comprehensive database of astronomy and physics publications maintained by the Smithsonian Astrophysical Observatory in Cambridge, Massachusetts, augmented with information from the preprint server arXiv.

The trio analysed almost 150,000 articles that were published in 5 major astronomy journals between 1950 and 2015. They found that the percentage of papers with a female first author rose from less than 5\% in the 1960 s to about $25 \%$ in 2015 . But since 1985 , astronomy publications with a male first author have received about $6 \%$ more citations than those led by a woman - a figure that Caplar and his co-authors suspected could reflect a hidden gender bias.

To determine the expected number of citations for publications led by women, the team trained an algorithm using nongender-specific data - including the seniority of the paper's first author and other criteria - that were derived from publications with male first authors. They then used the algorithm to predict the number of citations for papers with female first authors and compared this to the actual number the papers had collected.

Caplar notes that the trio's study cannot determine the drivers of gender bias. But if women leave the field in greater numbers or earlier in their career than do men, for example, they would not be in a position to present their findings at conferences or otherwise promote their papers in ways that could boost citations. 